\title{
Keep your interoceptive streams under control: An active inference perspective on anorexia nervosa
}

\author{
Laura Barca $^{1} \cdot$ Giovanni Pezzulo ${ }^{1}$ \\ Published online: 7 February 2020 \\ (C) The Psychonomic Society, Inc. 2020
}

\begin{abstract}
Anorexia nervosa (AN) is a psychiatric disorder primarily characterized by "restriction of energy intake relative to requirements leading to a significantly low body weight in the context of age, sex, developmental trajectory, and physical health" (DSM-5, n.d.). Here, we propose a novel interpretation of food restriction of AN, which elaborates on recent accounts of the brain as a predictive machine, continuously inferring and controling incoming signals, including bodily signals - i.e., interoceptive (active) inference. In this perspective, the extreme eating restrictions characterizing AN may serve the fundamental role of keeping under control (i.e., reducing excessively high levels of) interoceptive uncertainty, above and beyond controlling and mitigating concerns for body weight. In other words, noisy interoceptive streams may instantiate active strategies (i.e., eating restrictions) that amplify autonomic hunger signals, to minimize interoceptive uncertainty and maintain a more coherent sense of (interoceptive) self.
\end{abstract}

Keywords Anorexia nervosa $\cdot$ Interoception $\cdot$ Active inference $\cdot$ Uncertainty $\cdot$ Starvation $\cdot$ Self-coherence $\cdot$ Cognitive control

\section{Introduction}

Eating disorders, and in particular anorexia nervosa (AN), are widespread in the Western world. Despite extensive research, the mechanisms underlying AN and its striking eating restriction have still not been elucidated. AN is defined as "a syndrome in which the individual maintains a low weight as a result of a preoccupation with body weight, construed either as a fear of fatness or pursuit of thinness" (National Collaborating Centre for Mental Health (UK), 2004).

Several theories of AN thus focus on the psychological mechanisms responsible for the excessive concern for body weight. Cognitive theories of anorexia, as well as phenomenological reports from anorectic patients (Nordbø, Espeset, Gulliksen, Skårderud, \& Holte, 2006), suggest that the "need for control" of body size or weight might be central to eating restriction. In this perspective, AN patients would instantiate a series of "strategies" that keep their body size and weight under control, possibly to comply with externally defined esthetic standards.

Laura Barca

laura.barca@ istc.cnr.it

1 Institute of Cognitive Science and Technologies, ISTC-CNR, Via San Martino della Battaglia, 44, 00141 Rome, Italy
However, other psychological accounts of AN move beyond the centrality of concerns for body size and weight, and suggest the importance of investigating additional explanations for the fact that AN patients exert control over their bodies. For example, the control-based theory of anorexia posits that eating restriction acts as a mechanism to compensate for a sense of ineffectiveness and a lack of self-control. In other words, for anorectic patients, exerting control over their bodies would be motivating and rewarding per se, as it signals high efficacy (Fairburn et al., 1999; Fairburn \& Harrison, 2003; Froreich et al., 2016), rather than being a way to control body size and weight. Since self-efficacy relates to the ability to produce (desired) outcomes with sufficient certainty, this theory also suggests that anorectic patients may have problems dealing with excessive (outcome) uncertainty. In keeping with this, a growing body of empirical research suggests that difficulties in tolerating uncertainty might be central to the alteration of eating behaviors. The construct of "Intolerance of Uncertainty" - a predisposition to negatively perceive and respond to uncertain information and uncertain situations (Ladouceur et al., 2000) - is now considered a transdiagnostic vulnerability factor of different pathologies (Kesby et al., 2017) and of emotional disorders on the whole (Shihata et al., 2016). Compared to healthy controls, individuals with eating disorders have higher levels of intolerance of uncertainty as measured with a validated scale (Buhr \& Dugas, 2002), 
self-report symptoms description (Barca \& De Marchis, 2018), and are more distressed after the completion of a probabilistic task with varying levels of difficulty and uncertainty (Sternheim et al., 2011). Additionally, among individuals with disordered eating, anorectic patients had higher levels of intolerance of uncertainty than bulimic patients (Sternheim et al., 2011). A strong correlation between ratings of intolerance of uncertainty and alexithymia has been reported (Abbate-Daga et al., 2015), suggesting a possible role of this dimension on socio-emotional dysfunctions occurring in anorectic patients.

Other psychological theories point to the idea that in anorectic patients, the intolerance of uncertainty may be specific (or at least more pronounced) for bodily and emotional processing, with significant effects for the sense of self and for social interactions. Constructivist theories, for example, suggests that the emotional dimension of anorectic patients would be characterized by ambiguous feeling states elicited during (real or perceived) aversive social interactions. These feelings cannot be integrated within the diachronic narrative of the self and cause the perception of a loss of internal self-coherence (Mahoney \& Granvold, 2005). Similarly, it has been proposed that a deficit in cognitive structures related to personal identity might be the central feature of $\mathrm{AN}$, leading to a vague and indefinite perception of the self (Guidano, 1987). Recent empirical evidence provides some support for the hypothesized deficits in the socio-emotional dimension of anorectic patients. For example, in sentence-completion tasks describing ambiguous social scenarios, anorectic patients exhibit a negative bias (i.e., a higher number of negative interpretations) with respect to controls (Cardi et al., 2017). Other studies with anorectic patients report a misperception of social cues (Ambwani et al., 2016) and a diminished communication of (positive) emotions via facial expression (Leppanen et al., 2017).

In sum, the body of literature reviewed above suggests that concerns with uncertainty, and the need to control it, may be key to understanding eating disorders and in particular AN. While the sources of uncertainty are potentially plentiful, in the case of AN the most relevant ones might be specifically related to bodily events; which in turn may have cascading effects across multiple domains (emotional, social, and related to the self). Following this line of reasoning, we advance a novel proposal to explain how food restriction in AN may help dealing with uncertainty, and specifically with uncertainty arising from bodily information (i.e., noisy interoceptive processing and ambiguous feeling states) and the ensuing low self-coherence. The key idea that we advance is that food restriction can be used as an intentional - yet unconscious strategy to control (minimize) interoceptive uncertainty, above and beyond controlling body size and weight.

In the following sections, we further specify our proposal by linking it to both empirical evidence and formal theories of brain function. First, we review the recent literature on the processing of bodily and interoceptive information in $\mathrm{AN}$, which provides some background to the idea that dealing with excessive bodily (interoceptive) uncertainty may be a central concern in AN. Second, we briefly review a formal perspective on brain function - Active Inference - and explain how its mechanisms permit formulation of existing accounts of AN and its associated deficits. Third, we use Active Inference to introduce our novel proposal and the idea that starvation may be used to keep the excessive interoceptive uncertainty under control - and this, in turn, produces several maladaptive consequences, as the striking behavioral manifestation characterizing AN.

\section{Bodily and interoceptive processing in anorexia nervosa}

Bodily information is central to understanding our own emotional experiences, and part and parcel of "higher-order" perceptual and cognitive processes (Craig, 2003; Pezzulo et al., 2015a; Seth, 2013; Seth et al., 2012; Yu et al. 2020). The information that anorectic patients gather from their bodies appears compromised in many ways, with relevant implications for their emotional and social processing. For example, severe disturbances in body representation are well documented in AN (Gadsby, 2017; Keizer et al., 2013; Riva \& Gaudio, 2018; Spitoni et al., 2015). A body representation is an abstract perceptual representation of different characteristics of the body (i.e., its shape, the size of the body parts, and their position in space) and their integration into a whole entity. The cortical representations of our body and its parts are continuously updated by experiences gathered by different senses, as shown by its malleability to congruent multisensory stimulations. Thus, if the tactile stimulation of the hand (hidden from sight) is carried out in visual congruence on that of a fake hand, it can create an effect of incorporation of the fake hand and misallocation of the real hidden one, the so-called "rubber hand illusion" (Botvinick \& Cohen, 1998). Tactile stimulation is often used as an implicit measure of body representation (Keizer et al., 2011); and applied to AN patients, it revealed that their bodily distortion is not generalized but rather specific to the horizontal plane, with wider representations of horizontal tactile stimuli compared to vertical ones (Spitoni et al., 2015). This altered body representation is so pervasive that its effects go beyond perception, affecting body-scaled action, so that when asked to pass through a door-like opening, anorectic patients take a shoulder angle greater than their current body-size, but in line with their size overestimation (Keizer et al., 2013). However, healthy women with low self-esteem and body-image concern perform similarly to anorectic patients in an egocentric motor-imagery task, questioning the specificity of distorted body image to AN (Irvine et al., 
2019). Sensorial information gathered from the skin (e.g., the sense of touch) is also altered. Specifically, the perception of a particular type of touch - affective touch - appears to be reduced in individuals with AN, with reduced pleasantness specifically associated with slow stimulation of unmyelinated tactile C (CT) afferents (Crucianelli et al., 2016).

A growing body of evidence indicates that "inner-body" (i.e., interoceptive) information appears to be altered in anorexia. Interoception is the processing of afferent signals from internal organs of our body (e.g., stomach and bowels, heartbeat and breathing) leading to the perception and adaptive regulation of drive states, such as hunger and thirst, and of somatically relevant states such as effort and fatigue levels. In addition, interoceptive processing is considered to be key to the correct processing of emotional processes, of a sense of bodily self and of self-coherence (Craig, 2010; Seth \& Tsakiris, 2018). In keeping, there is a growing consensus that interoceptive and visceral signals specifically related to eating (e.g., incoming signals from the gastrointestinal tract) may be particularly important for cognition, emotion, consciousness, and the definition of a subject-centered frame of reference (Azzalini et al., 2019); and all the above processes may be impaired if interoceptive streams are corrupted. There is a growing consensus in connection various interoceptive deficits with anorexia nervosa (see Badoud \& Tsakiris, 2017, for a review). The discrimination between sensations and feelings, and between hunger and satiety, is compromised in AN (Fassino et al., 2004). This might be (at least partially) related to alteration in gastrointestinal motility. (Bluemel et al., 2017) measured postprandial gastrointestinal motor and sensory functions (i.e., gastric volumes, antral motility, breath samples, and sensations scores) at regular time intervals, in patients with disordered eating. AN patients had slower gastric emptying than obese and control participants and increased visceral sensation of fullness and lower sensation of hunger than the other groups. Interestingly, such differences decreased after a weight rehabilitation program, suggesting that they might be secondary to eating habits and psychiatric comorbidities.

The above studies suggest that AN is associated with impairments of interoceptive processing, but a comprehensive empirical assessment of these impairments remains elusive. One reason is that different facets of interoceptive processing are often discussed and measured in different ways, e.g., in terms of interoceptive accuracy (i.e., the accurate perception of interoceptive signals, sometimes measured with heartbeat counting), awareness (i.e., the meta-cognitive awareness of interoceptive accuracy, or the confidence in one's own interoceptive accuracy), and sensibility (i.e., the self-evaluated interoceptive belief, measured via questionnaire), and the recently developed concept of interoceptive predictive error, gathered from the difference between interoceptive accuracy and interoceptive sensibility measures (Young et al., 2017).
These different aspects of interoception are dissociable and differently related to eating behavior. For example, emotional eaters have higher interoceptive accuracy but lower metacognitive insight onto their interceptive abilities (Young et al., 2019). Emotional eating was also associated with higher interoceptive prediction error, which, in keeping with Garfinkel et al. (2016), has been interpreted as resulting from a failure to correctly incorporate afferent "bottom-up" interoceptive signals with top-down predictions. In the case of $\mathrm{AN}$, interoceptive accuracy and sensibility are both altered (Pollatos et al., 2008) and differently prone to change through therapy (Fischer et al., 2016). Additional support for the idea of altered (central) interoceptive processing in AN comes from functional imaging studies of remitted AN participants, demonstrating the increased activation of the right anterior insula during the anticipation and processing of pain stimuli, and interpreted as the suppression of the physiological feedback of pain (Strigo et al., 2013; see also the disconnection model of the insula of Nunn, Frampton, Fuglset, TörzsökSonnevend, \& Lask, 2011).

In sum, we have reviewed recent evidence indicating that AN patients may experience a fundamental deficit of interoceptive processing, which manifests in highly uncertain interoceptive and bodily information, plausibly magnified in the visceral and gastric domains. The excessively high level of bodily and interoceptive uncertainty may pose fundamental challenges for the formation of an accurate body representation as well as for the adaptive regulation of physiological needs, emotional processing, social interaction, and a unitary sense of (bodily) self. It is exactly these kinds of challenges that - we propose - AN patients may strive to avoid, by selecting behaviors such as starvation that are apparently maladaptive but at least permit exerting some control over interoceptive streams, thus potentially contributing to restoring more appropriate bodily and self models.

To explain how eating restrictions may play a role in controlling interoceptive uncertainty in $\mathrm{AN}$, we appeal to Active Inference, a formal framework that explains goaldirected behavior and cognition from the perspective of a predictive brain (Friston, 2010; Friston et al., 2017; Pezzulo et al., 2015b). Active Inference has been widely used to explain various psychopathological conditions, such as symptoms of perception, schizophrenia, psychosis, hysteria, and more (Adams et al., 2013; Corlett et al., 2019; Edwards et al., 2012; Paulus, 2019; Paulus et al., 2019a; Pezzulo et al. 2019a; Pezzulo et al. 2019b; Powers et al., 2017; Sterzer et al., 2018). Below, we briefly summarize the main constructs of Active Inference that are central to our proposal and illustrate how they may help to conceptualize AN-related deficits, as conceptualized in different theories that emphasize the control of body weight and size, intolerance of uncertainty, and the control of uncertain interoceptive streams (our proposal). 


\section{An active inference perspective on anorexia nervosa and its deficits}

Active Inference assumes that the brain uses an internal (generative) model to continuously infer and control exteroceptive signals coming from the external environment, proprioceptive signals associated witho our body movements and interoceptive signals from inside the body, e.g., from our internal organs. It does so to achieve the overall goal of $\mathrm{min}$ imizing prediction error (more formally, free energy) or the difference between (top-down) predictions from the generative model and (bottom-up) sensory signals from the periphery (Friston, 2010). This can be accomplished in two ways. First, by updating descending predictions to make them more similar to lower levels of sensory signals (i.e., changing one's mind about what one is perceiving). Second, by performing actions that change sensory samples and make them more similar to the predictions previously generated (e.g., changing what one is perceiving, as in the case of saccadic eye movements in visual perception). These two processes are called perceptual inference and Active Inference, respectively (Friston et al., 2017).

Importantly, the role prediction errors have in perceptual inference and active inference depends on their quality or precision (formally, an inverse variance): the most informative (high precision) signals are more weighted and havev a greater influence on the computations, whereas uninformative (low precision) signals are less weighted or ignored. The precision-weighting mechanism is key for balancing appropriately prior beliefs and sensory stimuli, as the signals that are assigned more (less) precision change prior beliefs to a greater (lesser) extent. Failures in precision-weighting have been associated with various pathological conditions. If prediction errors are incorrectly assigned a low precision, prior beliefs will dominate the inference and will not be updated appropriately. This might produce misperceptions, including "false symptoms" (Henningsen et al., 2018; Van den Bergh et al., 2017) or psychosis (Sterzer et al., 2018). Prior beliefs might also conform to aversive or highly unpredictable social interactions, as suggested for depressive mood (Badcock et al., 2017).

\section{Keeping body weight and size under control from an Active Inference perspective}

As noted above, in Active Inference, prior beliefs are important not just for perceptual inference but also for action selection and control - as they can play the role of goals (or setpoints), i.e., variables that the agent will try to keep under control. Normally, Active Inference balances the benefits and costs of various, hierarchically organized goals (e.g., higher social and cognitive and lower homeostatic goals) rather optimally, ensuring that behaviors having maladaptive consequences (e.g., starvation) are not selected (Pezzulo et al., 2018b). However, under certain conditions, these goals may fail to be correctly balanced, because (as in the perceptual examples above) some of them may enjoy excessively high precision. For example, an excessively precise prior belief about which body size is to be pursued (e.g., a very thin body) might determine the choice of eating restrictions that would eventually make this state of affairs true, down tuning other, undesired (e.g., homeostatic) consequences, which would fail to be sufficiently attended to, or integrated, during action selection. In sum, in Active Inference, maladaptive behavior associated with psychiatric syndromes including AN may be due to the failure to appropriately balance (the precision of) goals at different hierarchical levels - e.g., higher-level cognitive goals such as complying with some esthetic canon, or of "being in control," versus lower level drives such as maintaining a correct homeostasis or allostasis (Pezzulo et al., 2015b; Stephan et al., 2016; Sterling, 2012). Under certain maladaptive circumstances, these priors might not only enjoy too much precision but also fail to be correctly updated over time (e.g., while the person keeps losing weight), hence becoming inflexible and leading to continuance of inappropriate behavior. This schematic description provides an example of the ways Active Inference may help to conceptualize cognitive accounts of AN deficits that focus on patients' excessive concerns with body weight.

\section{Intolerance of uncertainty from an Active Inference perspective}

Active Inference may also help conceptualize the "intolerance of uncertainty" associated with AN - which is the main focus of this article. During action selection, Active Inference automatically balances two factors: an economic (or extrinsic) value, associated with goal-directed behavior and the achievement of rewarding states; and an epistemic (or intrinsic) value, associated with information-seeking behavior and the reduction of uncertainty (Friston et al., 2015). This implies that Active Inference constantly balances exploitation and exploration; and depending on contextual conditions, it may favor action plans that pursue economic value (i.e., yields the highest reward) or epistemic value (i.e., yields the highest information gain), or a combination of both. Interestingly, there are situations in which the action plan that yields the highest reward cannot be selected with sufficient confidence until one has reduced uncertainty first. For example, one may not be able to judge whether or not to attend a party, until he/ she is sufficiently certain about who else has been invited (e.g., whether or not he/she will meet somebody undesired). In these situations, Active Inference mandates the selection of action plans whose first (epistemic) part resolves the 
uncertainty (e.g., try to know who was invited) and whose second part makes the choice on the base of the novel information (e.g., decide whether or not to attend, depending on who else was invited)

The inference leading to the balance of epistemic, uncertainty-minimizing versus economic, rewardmaximizing action plans can, however, become maladaptive, for several reasons. Normally, an Active Inference agent would consider the quality of its information sources in the decision about whether or not to attend to them and immediately ceases to gather imprecise information or information that has no epistemic value or information gain (Parr \& Friston, 2017). However, a person having excessive levels of uncertainty-intolerance (e.g., excessively high and inflexible prior beliefs about the precision of his/her information sources) may indulge in excessive attempts to reduce his/her uncertainty even when this is very costly or impossible. In the long run, these failed attempts to reduce uncertainty may lead to beliefs of low self-efficacy, when the latter is evaluated against standards that are excessively high.

Furthermore, a person excessively concerned with reduction of uncertainty may put into place strategies that would reduce uncertainty in the short term, but can be maladaptive in the long term. Imagine a person who is organizing a party and has some uncertainty about, for example, how the party will unfold in time, and whether it will be appreciated. She can reduce this uncertainty by specifying all the details of the party - until she is confident enough that the party will meet her expectations. However, like any other real-world problem, organizing a party entails multiple sources of uncertainty, some of which may be difficult or impossible to reduce hence requiring the person to deal with situations that entail some residual uncertainty and risk of failure. If the person is excessively concerned with reduction of uncertainty, she may indulge in compulsive attempts to control environmental factors that are either irrelevant or beyond her reach. Another maladaptive strategy to keep uncertainty under control consists of performing repetitive actions, as they diminish the uncertainty (and entropy) of action outcomes - at the cost of having poor adaptive value. Performing repetitive behaviors is a defining trait of obsessive-compulsive disorder, which has a high co-morbidity with eating disorders, suggesting a potential trans-diagnostic vulnerability to uncertainty. Finally, one may also momentarily deal with uncertainty by learning internal models that are poorly differentiated, (e.g., models that use a single variable to explain everything and hence can assimilate virtually any stimulus - at the cost of being almost useless) and by reducing his/her exposition to contexts that are by definition more uncertain, such as novel contexts. The problem with all these strategies is that while they may succeed in reducing uncertainty in the short term, they are degenerate solutions deprived of long-term adaptive benefits (and hence, under normal conditions, avoided in Active Inference).
Furthermore, these degenerate solutions all reduce drastically epistemic and exploratory behavior, which is instead mandatory for an Active Inference agent to learn adaptive models (Friston et al., 2015; Tschantz et al., 2019). In other words, these strategies all prevent agents from experiencing events that bring information gain and are important for learning to control efficaciously their environment - thus pushing the agents into a small, controllable corner of their ecological niche (e.g., in a dark room (Friston et al., 2012)).

\section{Interoceptive processing and starvation from an Active Inference perspective}

So far, we discussed how Active Inference may provide a mechanistic ground to various theories of AN, which emphasize excessive concerns with body weight and size or intolerance of uncertainty. Here, we expand the analysis of the latter point - intolerance of uncertainty - to the interoceptive domain, in order to introduce our novel proposal on the starvation symptom of AN, which we conceptualize as a way to keep the uncertainty of interoceptive streams under control. For this, we first need to briefly introduce the Active Inference perspective on interoceptive inference and its deficits.

Active Inference applies not just to the estimation and control of external states, but also to the estimation and control of interoceptive streams (e.g., heartbeat, visceral organs) and physiological processes - which are key for autonomic regulation, emotional experience (Iodice et al., 2019; Pezzulo, 2013; Pezzulo et al., 2018b, 2015b; G. Pezzulo et al., 2018), the construction of the self (Seth, 2013), and the emergence of pathological conditions (Barrett et al., 2016; Friston et al., 2014; Murphy et al., 2017; Paulus et al., 2019a; Quadt et al., 2018). According to theories of interoceptive inference (Seth, 2013; Seth \& Friston, 2016) and Embodied Predictive Interoception Coding (EPIC) (Barrett \& Simmons, 2015), interoceptive processing uses the same constructs of Active Inference described above for perception and action. In this perspective, interoception is not a purely bottom-up process but critically engages brain predictions about the inner state of the body. Descending interoceptive predictions about bodily states play a key regulatory role, as they engage autonomic reflexes (an example of internally directed Active Inference) while ascending interoceptive signals inform and update these predictions, generating a circular causality. The mismatch between predictions and sensory signals creates interoceptive prediction errors, which are precision-weighted in the same way as exteroceptive prediction errors.

This Active Inference account of interoceptive inference provides the starting point of our explanation of the starvation behavior characterizing restrictive $\mathrm{AN}$. We propose that a key factor in the etiology of AN is a specific deficit of interoceptive processing, with interoceptive streams that are 
excessively noisy (i.e., have low signal-to-noise ratio). Noisy interoceptive streams are dangerous for homeostatic control as they can entail poor perception (or misinterpretation) of bodily states (Petersen et al., 2014) and prevent the development of accurate and well-differentiated internal models, e.g., models that correctly distinguish signs of low versus high hunger, thirst, and fatigue. Furthermore, if one assumes that interoceptive processing is key to the formation of self models (Seth, 2013), then noisy interoceptive streams may lead to a diminished sense of central coherence and a blurred perception of the self (Fig. 1, panel a).

Noisy interoceptive streams may also significantly impair one's ability to successfully engage in social interactions. In these situations, AN patients may experience streams of uncertain feeling states, rendering them unable to correctly infer "social causes" for the observed behavior (e.g., is this person friendly or not?), hence rendering the whole social situation aversive. In turn, this would produce a further lack of internal coherence, as the social experiences and the ensuing emotions cannot be easily integrated within a diachronic narrative of the self. Finally, over time, Active Inference would also assign noisy interoceptive streams a low precision (because, as noted above, attending them does not reduce uncertainty); and this entails the person progressively devoting less and less attention to his/her bodily signals, thus preventing the correct updating of bodily and self models. In sum, noisy interoceptive streams may induce a cascade of problems that span immediate adaptive action selection, the construction of bodily, social, and self models. Starvation may stem from an attempt to remedy this situation: it may be selected as a purposive regulatory behavior aimed at reducing interoceptive uncertainty.

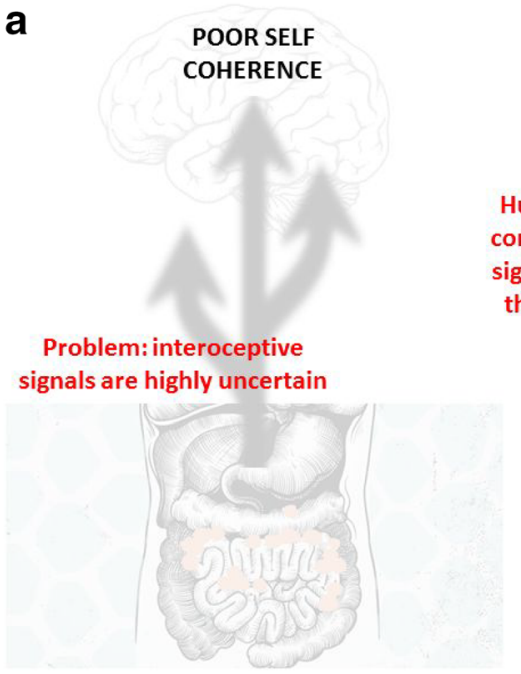

Fig. 1 Schematic illustration of the hypothesis that starvation may serve to control the uncertainty of interoceptive streams in anorexica nervosa (AN) patients. Panel A: AN patients receive noisy interoceptive information from their "inner bodies" and thus cannot correctly discriminate them or form well-differentiated interoceptive generative models. In the
In this perspective, individuals with AN might use food restrictions to actively amplify their interoceptive signals of hunger (Fig. 1, panel b) hence reducing at least in part their excessive uncertainty. In Active Inference, the reduction of uncertainty would entail short-term benefits, of at least two kinds: first, it might increase the epistemic value (and information gain) of interoceptive information; second, it might have some hedonic effect, under the assumption that prediction error minimization is associated with positive affect (Joffily \& Coricelli, 2013). The reduction of uncertainty may entail longer-term benefits, too, by helping restore a more precise and coherent model of bodily signals and sense of self and more adaptive homeostatic and allostatic loops, providing some relief from the distress of allostatic misregulations and inefficacy. These advantages - all of which are related to the epistemic dimension of Active Inference - may be responsible for the (seemingly paradoxical) maintenance egosyntonic nature of the disorder (Gregertsen et al., 2017), above and beyond the pragmatic goal of maintaining a low body weight.

Yet, using starvation as a way to reduce interoceptive uncertainty has severe consequences. The most obvious ones relate to maladaptive homeostatic regulation and loss of body weight and size, but there is more than that. The artificial increase of the precision of interoceptive signals may only partially solve the problem of uncertainty, especially if there is some fundamental deficit that renders interoceptive streams excessively noisy. Consider, as a rough analogy, a situation in which one raises the volume of a radio transmitting a mixture of a song and background noise - and both the signal (the song) and the noise levels increase. Such situations may impair the development of correct bodily models.

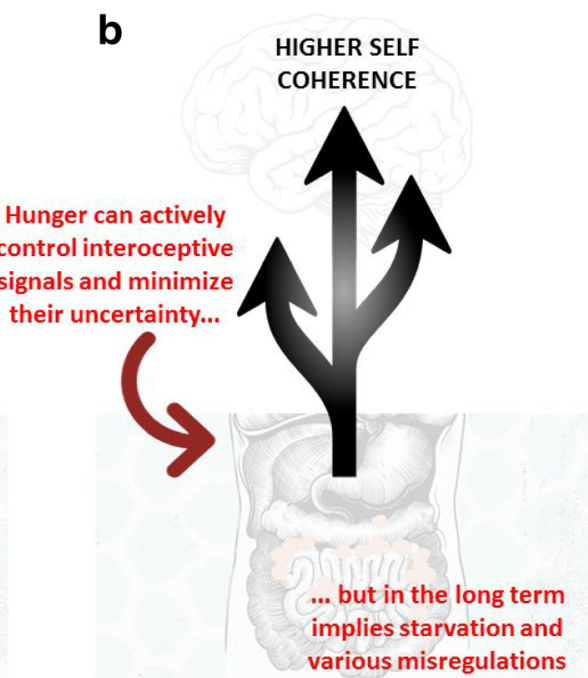

long run, this may result in a blurred perception of the self (or poor bodyand self-models). Panel B: AN patients may strategically use starvation to actively amplify their interoceptive signals, making them less uncertain and contributing to restore a precise and well-differentiated model of the body and the self 
Furthermore, the overuse of repetitive strategies (like starving) prevents the generation of variable outcomes that bring information gain and are therefore essential for the correct learning and update of internal models (Tschantz et al., 2019). This comes at the risk of rendering bodily models poorly differentiated, inflexible, and ultimately detached from reality. With time, precision computations in AN may become divorced from optimality (i.e., signal-to-noise ratios), too, and assign excessively high precision to some interoceptive signals (e.g., gastric signals) even when they remain noisy. When these signals enter in the multisensory integration that is necessary to estimate somatic and homeostatic variables, they may be incorrectly weighted more than other signals (e.g., pain, touch) that are potentially more reliable. Not only this may produce disorders of body perception but also disorders of homeostatic regulation, which in interoceptive inference theories is based on a correct estimate of somatic and homeostatic variables (Seth \& Friston, 2016).

In sum, we propose that the active control of interoceptive signals via food restrictions may be a key mechanism in the etiology of AN. In individuals with AN, starvation may be actively used to reduce interoceptive uncertainty to acceptable levels, also potentially contributing to the development of less fragmented models of bodily signals and of the self. This strategy also has several maladaptive consequences but is not "irrational" from the perspective of an Active Inference agent that puts a premium on low levels of uncertainty. Active Inference may help to shed light on the most paradoxical aspect of starvation in AN: the fact that it is maintained, despite it preventing correct homeostatic processes that constitute the most important pragmatic imperatives for living organisms. In Active Inference, there are epistemic imperatives (e.g., lowering hidden state uncertainty) that are equally important as pragmatic imperatives - and that under specific maladaptive conditions may become dominant.

We believe that our proposal provides new insights for both guiding empirical research and refining computational models to unveil the mechanisms underlying the complexity of AN. At the same time, it raises important interrogatives that remain to be investigated in future research. An open question is whether individuals with AN have specific deficits with regard to hunger-related signals or broader interoceptive signals; and in the latter case, why do they actively control hunger signals, as opposed to other interoceptive signals. A possible explanation is that actively controlling hunger sensations by limiting food intake may be much simpler than controlling other internal bodily signals - which is in general not straightforward. This would imply that despite AN being an eating disorder, it may not be necessarily (or exclusively) related to the eating domain. Indeed, the active control mechanism discussed here might also account for the co-occurrence of self-injury behavior in some individuals with AN (Claes et al., 2015).
Another related question is under which conditions the necessity to keep interoceptive uncertainty under control leads to AN. Indeed, there are various ways to control interoceptive uncertainty - and even specifically noisy gut sensations besides starvation. In principle, one can obtain more precise hunger-related sensations by eating more (to produce a constant "satiety signal") rather than by starving (to produce a constant "hunger signal"). The choice of which specific behavioral strategy to use to reduce interoceptive uncertainty (e.g., starving vs. excessive food consumption, possibly leading to forms of obesity) may depend on prior preferences (e.g., for a certain body weight or size). This perspective may help reconcile our view with cognitive theories that emphasize concerns for body weight and size in AN patients: these concerns may be amongst the driving factors that determine the choice of starvation as the preferred strategy to reduce interoceptive uncertainty.

Yet another research question is whether, and to what extent, the basic mechanisms of homeostatic and allostatic regulation are impaired in individuals with AN. In general, in Active Inference, homeostatic drives and goals correspond to very precise priors at some deep hierarchical level, which generate top-down predictions about (for example) gut and other signals indicating satiety (Pezzulo et al., 2015b). When one experiences interoceptive signals incompatible with such predictions (i.e., gut signals indicating that one is not satiated) a precise prediction error is propagated upward in the hierarchy that needs to be minimized. Since the above survivalrelated priors are very precise and hence virtually cannot be revised, the only way to minimize prediction error is by feeding - to make the interoceptive signal more similar to the prior prediction. In our proposal, we emphasized that individuals with AN may experience imprecise interoceptive signals and prediction errors, which would render their estimate of interoceptive state more uncertain and their homeostatic and allostatic regulation less adaptive. It remains to be investigated in future research whether other aspects of regulatory interoceptive loops may be impaired in AN individuals; for example, whether the precision of their priors is misregulated and whether the imprecise interoceptive streams depend on fundamental physiological problems with interoceptive channels or misregulations of precision computations.

\section{Discussion}

We proposed a novel account of the most striking manifestation of anorexia nervosa (AN): starvation. In this account, food restriction is a strategy to deal with high uncertainty within interoceptive streams. By limiting food intake until starvation, AN individuals maximize the gain of their autonomic signals of hunger, thus reducing (to some extent) their interoceptive uncertainty and possibly regaining their sense of 
self-coherence and self-control - at the expense of possible homeostatic misregulations.

Our proposal is fundamentally different from previous accounts of $\mathrm{AN}$, as it posits that eating restrictions stem from an adaptive strategy to deal with excessive interoceptive uncertainty, above and beyond the control of body size and weight. In Active Inference terms, starvation would serve the epistemic imperative to keep (interoceptive) uncertainty under control - at the expense of another, pragmatic imperative to maintain a correct homeostasis.

Another important aspect that distinguishes our proposal from previous accounts is our conceptualization of AN as a disorder of interoceptive processing and of the self, rather than merely of body image (see also Amianto, Northoff, Abbate Daga, Fassino, \& Tasca, 2016). Indeed, we argue that (noisy) afferent bodily signals are central to the development of the pathology, and subsequently contribute to its maintenance. In this perspective, our proposal aims to combine the computational-level constructs of Active Inference (and interoceptive inference within it) with psychological-level theories of cognitive control and self-coherence; and more specifically, with a constructivist perspective that grounds self-models into interoceptive streams and consider the extreme need to control eating to be the key maintaining factor of AN (Fairburn et al., 1999; Surgenor et al., 2002).

There are several lines of evidence supporting our Active Inference account of AN. Individuals with AN show high levels of alexithymia and neurobiological markers of interoceptive dysregulation (Kessler et al., 2006; Strigo et al., 2013), raising the possibility that they have an impaired ability to access or process interoceptive information. The altered distribution of fixation patterns associated with the estimation of body-size (Cornelissen et al., 2016) might be evidence of a cooccurring failure in actively sampling perceptual information to reduce sensory uncertainty. Furthermore, the tendency of AN patients to evaluate themselves from a third-person perspective, or "self-objectification" (Dakanalis et al., 2017), might be a vicarious mechanism that uses visuo-perceptual information to compensate interoceptive (Pollatos et al., 2008) and central coherence deficits (Hamatani et al., 2018).

Interoceptive models of disordered eating have been described in the literature (see Klabunde et al., 2017, for such an account of bulimia nervosa), but this is the first model that specifically focuses on the etiopathological mechanisms of AN. Interestingly, in parallel to our proposal, a recent review of the trans-diagnostic role of interoception in psychiatric disorders briefly mentions the case of $\mathrm{AN}$; and specifically that "AN patients may have context-specific (e.g., hunger state) hyper-precise priors that generate strong expectations about visceral sensations. Importantly, it may be the selection of particular actions in response to these hyper-precise priors (e.g., starvation to avoid the actual sensing of visceral sensations) that forms one core feature of the psychopathology"
(Paulus et al., 2019b). While Paulus et al.'s and our accounts start from a similar conceptual framework, they provide substantially different explanations of AN deficits (i.e., correct sensations but hyper-precise priors vs. high interoceptive uncertainty) and of starvation (i.e., avoidance vs. active amplification of visceral sensations). At this stage, both proposals are speculative and additional evidence is required to adjudicate amongst them - or to combine them. Indeed, it is possible that hyper-precise priors and high interoceptive uncertainty may root AN in different patient populations, reflecting the heterogeneity of the disorder. One possible way to distinguish between these possibilities would be stimulating the insular cortex of AN patients to modulate the precision of interoceptive signals, as recently described by (Sagliano et al., 2019) - as this manipulation should affect patients' interoceptive processing in our proposal, but not necessarily in Paulus et al.'s account (where interoceptive signals would be largely ignored, regardless of their effective precision). In principle, according to our proposal, restoring precise interoceptive signals might contribute to preventing or abolishing starvation (and other aspects of AN); but designing interoceptive manipulations that are efficacious on clinical populations may be problematic.

Another theoretical proposal that is related to ours is the idea that depression and persistent negative affective feelings stem from a misregulation of allostasis, which is (at least in part) caused by noisy (or otherwise unreliable) afferent interoceptive signals and prediction errors (Barrett et al., 2016). The co-morbidity between AN and depression suggests that uncertainty in interoceptive streams may be a trans-diagnostic trait in both. However, the attitude towards such uncertainty may be different in $\mathrm{AN}$ and depression: while some aspects of $\mathrm{AN}$, such as starvation (but also potentially compulsive behavior and self-injury), are better characterized as active strategies to minimize interoceptive uncertainty, depression seems to be associated with a more passive attitude, potentially leading towards a mental, and sometimes also a physical, withdrawal from the world (Barrett et al., 2016).

A prediction that stems from our proposal is that experimental manipulations that interfere with the correct cortical processing of visceral sensations (e.g., gastric sensations) in non-pathological individuals might impact their emotional processing, as measured for example using topographical bodily representations (Khalsa et al., 2018; Nummenmaa et al., 2014), as well as the execution to body-scale action (Keizer et al., 2013). Similar effects may be produced with experimental manipulations that artificially increase the uncertainty of interoceptive channels. However, inducing interoceptive uncertainty (either centrally or peripherally) is challenging, especially given the tendency of the interoceptive system to maintain homeostasis for survival. One possibility might be to induce electrical interference at brain regions integrating bodily states, as the insula. In Pollatos et al. 
(2016) transcranial magnetic stimulation (TMS) over the anterior insula hampered the accurate perception of cardiac and respiratory signals. If we consider such declined perception of sensory signal as a proxy of interoceptive uncertainty, we would predict that a higher level of interoceptive uncertainty (induced, e.g., by TMS stimulation of anterior insula) in nonclinical populations might affect the recognition of one own and others' emotions, such as for example faces expressing different types of emotions (Pezzulo et al., 2018a; Yu et al. 2020) as well as performance in social decision-making tasks, given the centrality of the insular cortex in social dynamics (Rogers-Carter \& Christianson, 2019). Analogously, manipulations that target (increase interoceptive uncertainty of) other bodily and visceral sensations, including gastric sensations, may potentially induce (reduced and transient) forms of eating disorders. On the other hand, if an interoceptive deficit underlies the development of food restriction in $\mathrm{AN}$, then training aimed at reducing interoceptive uncertainty might have cascading effects on food restriction. (Boswell et al., 2015) suggest that "interoceptive exposure" techniques that were effective in the cases of anxiety and fear-related disorders might be useful in treating eating disorders, too. However, it is important to note that the basic understanding of which (or how many) of the different facets of interoception are compromised in AN needs to be prioritized in order to program effective treatments.

\section{Future directions}

One of the strengths of our proposal is that it is grounded in a general framework that describes formally the interactive dynamics of predictive and sensory signals in the brain: Active Inference. Our proposal deliberatively focuses on a specific mechanism - starvation behavior of the restrictive type AN and highlights its (paradoxical) adaptive benefits within an Active Inference scheme, which puts the minimization of interoceptive uncertainty at a premium. However, as reviewed in the Introduction, there are many alternative perspectives on AN (reflecting its complexity). Our proposal is not necessarily incompatible with but can be complementary to other proposals that focus on cognitive factors such as (for example) concerns with body size, culturally induced esthetic canons, or broad forms of compulsion and uncertainty-avoidance, even outside the interoceptive domain. These different proposals share some common features (as highlighted by our analysis in Active Inference terms) but focus on different, potentially complementary mechanisms for the acquisition and maintenance of AN. It remains to be clarified in future research which of these mechanisms is more fundamental for AN and how they give rise to the multiplicity of its symptoms. For example, there is no doubt that (a concern for) the body plays an important role in eating disorders; but while some theories consider this concern to be the driving force of $\mathrm{AN}$, others consider it to be manifestations of the need to restore central coherence and sense of a unitary self (see also Surgenor et al., 2002). As our knowledge of AN progresses, we will be able to better disentangle between these possibilities - or also combine them into more sophisticated formal models, as it is questionable whether one single mechanism would provide a complete account of multifarious psychiatric disorders such as AN.

A possible way to advance our understanding of the interoceptive system in clinical and non-clinical populations might be by using bodily and interoceptive illusions to determine whether and how different individuals organize their bodily sensations within a coherent "interoceptive schema" (Iodice et al., 2019). By systematically considering whether patient groups are sensitive or insensitive to different bodily and interoceptive illusions, it may be possible to map the specificities of their misperceptions. For example, studying to what extent anorectic patients are sensitive to full-body illusions created with visuotactile stimulation (Carey et al., 2019) and respiratory congruency (Monti et al., 2019) might shed light on the malleability of their hierarchical organization of interoceptive variables. The degree to which patients with disordered eating might be sensitive to such manipulations is not clear yet, as mixed results have been reported. In Carey et al. (2019), for example, participants with eating disorder vulnerability did not differ from controls in the degree of their embodiment, nor were differentially affected by affective and non-affective touch. But in Keizer et al. (2016) both synchronous and asynchronous tactile stimulation during full-body illusion enhanced the embodiment of an avatar, affecting also estimation of body size of anorectic patients. The specific (in) sensitivity of anorectic patients to bodily and interoceptive illusions remains to be systematically investigated.

Another important objective for future research is characterizing more precisely the developmental trajectory of AN. Intra-family factors likely play a central role in the development of a sense of self-coherence and of competence. Eating disorders might be rooted in (critical events undermining) the first stages of infant development, when individuals form models of their individual bodies and self, as differentiated from the rest of the world. Deficits of interoceptive processing during this developmental stage - either due to endogenous causes or as the consequence of being exposed to critical events - may impair the formation of accurate body and self models, which in turn may impair the correct identification of one's own emotions (e.g., alexithymia) and lower interoceptive sensitivity (Murphy et al., 2017). By having less precise and more malleable body-representations, patients with AN may be less able to form a coherent sense of self "from the inside" (as measured for example by the experience of changes in self-other boundaries in response to multisensory stimulation (Tajadura-Jiménez \& Tsakiris, 2014)), thus potentially increasing the 
importance of other (e.g., social) sources of information to define the self.

Finally, it is important to map more systematically theories of AN at different levels of analysis, which consider social, cognitive, neurobiological, physiological, and metabolic processes. For example, a comprehensive account of AN should clearly identify specific dysregulations of basic homeostatic, appetitive, and aversive systems, as mediated (at least in part) by neuromodulators such as dopamine and serotonin - a topic that is currently actively discussed (Kaye et al., 2013). Our explanation of the starvation symptom of AN does not necessarily require dysregulations of reward or punishment systems in the brain - as it may depend on the epistemic imperative or reducing uncertainty under Active Inference. However, extending our current model to also cover potential dysregulations of drive and reward systems and to consider the roles of neuromodulators is an important objective for future research. Furthermore, we foresee the future development of our model to extend its scope to metabolic and endocrinological dysfunctions, which are receiving increasing interest in relation to anorexia and other psychopathological conditions (Watson et al., 2019). This is in keeping with recent integrative accounts of psychopathologies that consider multiple dysfunctions at the cortical, neuroendocrine, psychological, and behavioral levels (see Gorwood et al., 2016, for a review). In the case of anorexia, it has been proposed that the disorder might be a maladaptive "biopsychosocial" response to interpersonal stress events rooted in early life experiences (Cardi et al., 2018) and may be coupled with genetic and biological factors. In this perspective, Connan et al. (2003) proposed that genetic factors and primary interpersonal/affective experiences (i.e., attachment style) jointly induce a dysregulation of the hypothalamic-pituitary-adrenal (HPA) axis and subsequent poorly regulated stress response. Aberrant HPA axis response induces (among other things) elevated release of corticotrophin-releasing hormone (CRH), which is associated with AN symptoms such as loss of appetite and weight. A more comprehensive model of AN should also focus on the important roles of metabolic hormones, such as ghrelin (originating from the gastrointestinal and pancreatic cells) and leptin (originating from adipose cells), which are both involved in appetite regulation and homeostasis (Wu \& Kral, 2004) and dysfunctional eating (Grzelak et al., 2017). These metabolic hormones are central to energy regulation in the brain and have been recently suggested to also play a role in the socio-affective dimension (MacCormack \& Muscatell, 2019). Typically, the plasma level of ghrelin is high before a meal (in signaling the need for food intake) and decrease after food consumption. Anorectic patients present a series of dysfunction in ghrelin expression, with higher levels of ghrelin and impaired post-prandial modulations (i.e., delayed or decreased after meal consumption) (see for Schalla \& Stengel, 2017, for a review). In the light of our model, high levels of ghrelin might be considered as an increase in sensory signal originated from the gut, but further research is needed to develop such a hypothesis.

\section{Conclusion}

In sum, neurobiological, cognitive, and social factors (among others) co-occur in the etiology and maintenance of AN probably, with a different weight depending on the developmental stage of the disorder - and an open objective for future research should assess their relations and relative importance. We believe that the adoption of a formal framework to refine the theoretical proposals and render their prediction more quantitative can be helpful, and hope that our illustrative examples using Active Inference may be a first step in this direction.

Acknowledgements We thank Matthew Sims for valuable discussions and comments on an earlier version of the article.

Author contributions LB and GP conceptualized the manuscript; LB drafted the manuscript; GP revised it critically for computational content; LB and GP provided final approval for publication.

\section{Compliance with ethical standards}

Conflict of interest The author declares that the research was conducted in the absence of any commercial or financial relationships that could be construed as a potential conflict of interest.

\section{References}

Abbate-Daga, G., Quaranta, M., Marzola, E., Amianto, F., Fassino, S., 2015. The Relationship between Alexithymia and Intolerance of Uncertainty in Anorexia Nervosa. Psychopathology 48, 202-208. https://doi.org/10.1159/000381587

Adams, R.A., Stephan, K.E., Brown, H.R., Frith, C.D., Friston, K.J., 2013. The Computational Anatomy of Psychosis. Frontiers in Psychiatry 4. https://doi.org/10.3389/fpsyt.2013.00047

Ambwani, S., Berenson, K.R., Simms, L., Li, A., Corfield, F., Treasure, J., 2016. Seeing things differently: An experimental investigation of social cognition and interpersonal behavior in anorexia nervosa. The International Journal of Eating Disorders 49, 499-506. https://doi. org/10.1002/eat.22498

Amianto, F., Northoff, G., Abbate Daga, G., Fassino, S., Tasca, G.A., 2016. Is Anorexia Nervosa a Disorder of the Self? A Psychological Approach. Frontiers in Psychology 7. https://doi. org/10.3389/fpsyg.2016.00849

Azzalini, D., Rebollo, I., Tallon-Baudry, C., 2019. Visceral Signals Shape Brain Dynamics and Cognition. Trends in Cognitive Sciences 23, 488-509. https://doi.org/10.1016/j.tics.2019.03.007

Badcock, P.B., Davey, C.G., Whittle, S., Allen, N.B., Friston, K.J., 2017. The Depressed Brain: An Evolutionary Systems Theory. Trends in Cognitive Sciences 21, 182-194. https://doi.org/10.1016/j.tics. 2017.01.005

Badoud, D., Tsakiris, M., 2017. From the body's viscera to the body's image: Is there a link between interoception and body image 
concerns? Neuroscience and Biobehavioral Reviews 77, 237-246. https://doi.org/10.1016/j.neubiorev.2017.03.017

Barca, L., Marchis, M.D., 2018. The case of Sofia: an example of the dynamic properties of the therapeutic relationship. 10.31219/osf.io/ $\mathrm{s} 3 \mathrm{~m} 6 \mathrm{p}$

Barrett, L.F., Quigley, K.S., Hamilton, P., 2016. An active inference theory of allostasis and interoception in depression. Philosophical Transactions of the Royal Society of London. Series B, Biological Sciences 371. https://doi.org/10.1098/rstb.2016.0011

Barrett, L.F., Simmons, W.K., 2015. Interoceptive predictions in the brain. Nature Reviews. Neuroscience 16, 419-429. https://doi.org/ $10.1038 /$ nrn3950

Bluemel, S., Menne, D., Milos, G., Goetze, O., Fried, M., Schwizer, W., Fox, M., Steingoetter, A., 2017. Relationship of body weight with gastrointestinal motor and sensory function: studies in anorexia nervosa and obesity. BMC Gastroenterology 17, 4. https://doi.org/ 10.1186/s12876-016-0560-y

Boswell, J.F., Anderson, L.M., Anderson, D.A., 2015. Integration of Interoceptive Exposure in Eating Disorder Treatment. Clinical Psychology: Science and Practice 22, 194-210. https://doi.org/10. 1111/cpsp.12103

Botvinick, M., Cohen, J., 1998. Rubber hands "feel" touch that eyes see [8]. Nature 391, 756. https://doi.org/10.1038/35784

Buhr, K., Dugas, M.J., 2002. The Intolerance of Uncertainty Scale: psychometric properties of the English version. Behaviour Research and Therapy 40, 931-945. https://doi.org/10.1016/s0005-7967(01) 00092-4

Cardi, V., Tchanturia, K., Treasure, J., 2018. Premorbid and Illnessrelated Social Difficulties in Eating Disorders: An Overview of the Literature and Treatment Developments. Current Neuropharmacology 16, 1122-1130. https://doi.org/10.2174/ 1570159 X16666180118100028

Cardi, V., Turton, R., Schifano, S., Leppanen, J., Hirsch, C.R., Treasure, J., 2017. Biased Interpretation of Ambiguous Social Scenarios in Anorexia Nervosa. European Eating Disorders Review the Journal of the Eating Disorders Association 25, 60-64. https://doi.org/10. 1002/erv.2493

Carey, M., Crucianelli, L., Preston, C., Fotopoulou, A., 2019. The Effect of Visual Capture Towards Subjective Embodiment Within the Full Body Illusion. Scientific Reports 9, 2889. https://doi.org/10.1038/ s41598-019-39168-4

Claes, L., Islam, M.A., Fagundo, A.B., Jimenez-Murcia, S., Granero, R., Agüera, Z., Rossi, E., Menchón, J.M., Fernández-Aranda, F., 2015. The Relationship between Non-Suicidal Self-Injury and the UPPS-P Impulsivity Facets in Eating Disorders and Healthy Controls. PLoS One 10, e0126083. https://doi.org/10.1371/journal.pone.0126083

Connan, F., Campbell, I.C., Katzman, M., Lightman, S.L., Treasure, J., 2003. A neurodevelopmental model for anorexia nervosa. Physiol. Behav., Proceedings from the 2002 Meeting of the Society for the Study of Ingestive Behavior (SSIB) 79, 13-24. https://doi.org/10. 1016/S0031-9384(03)00101-X

Corlett, P.R., Horga, G., Fletcher, P.C., Alderson-Day, B., Schmack, K., Powers, A.R., 2019. Hallucinations and Strong Priors. Trends in Cognitive Sciences 23, 114-127. https://doi.org/10.1016/j.tics. 2018.12.001

Cornelissen, K.K., Cornelissen, P.L., Hancock, P.J.B., Tovée, M.J., 2016. Fixation patterns, not clinical diagnosis, predict body size overestimation in eating disordered women and healthy controls. The International Journal of Eating Disorders 49, 507-518. https://doi. org/10.1002/eat.22505

Craig, A.D., 2003. Interoception: the sense of the physiological condition of the body. Current Opinion in Neurobiology 13, 500-505.

Craig, A.D.B., 2010. The sentient self. Brain Structure \& Function 214, 563-577. https://doi.org/10.1007/s00429-010-0248-y

Crucianelli, L., Cardi, V., Treasure, J., Jenkinson, P.M., Fotopoulou, A., 2016. The perception of affective touch in anorexia nervosa.
Psychiatry Research 239, 72-78. https://doi.org/10.1016/j. psychres.2016.01.078

Dakanalis, A., Clerici, M., Bartoli, F., Caslini, M., Crocamo, C., Riva, G., Carrà, G., 2017. Risk and maintenance factors for young women's DSM-5 eating disorders. Archives of Women's Mental Health 20, 721-731. https://doi.org/10.1007/s00737-017-0761-6

DSM-5 [WWW Document], n.d. URL https://www.psychiatry.org/ psychiatrists/practice/dsm (accessed 6.26.19).

Edwards, M.J., Adams, R.A., Brown, H., Pareés, I., Friston, K.J., 2012. A Bayesian account of "hysteria." Brain: A Journal of Neurology 135, 3495-3512. https://doi.org/10.1093/brain/aws129

Fairburn, C.G., Harrison, P.J., 2003. Eating disorders. The Lancet 361, 407-416. https://doi.org/10.1016/S0140-6736(03)12378-1

Fairburn, C.G., Shafran, R., Cooper, Z., 1999. A cognitive behavioural theory of anorexia nervosa. Behaviour Research and Therapy 37, 1 13.

Fassino, S., Pierò, A., Gramaglia, C., Abbate-Daga, G., 2004. Clinical, psychopathological and personality correlates of interoceptive awareness in anorexia nervosa, bulimia nervosa and obesity. Psychopathology 37, 168-174. https://doi.org/10.1159/000079420

Fischer, D., Berberich, G., Zaudig, M., Krauseneck, T., Weiss, S., Pollatos, O., 2016. Interoceptive Processes in Anorexia Nervosa in the Time Course of Cognitive-Behavioral Therapy: A Pilot Study. Frontiers in Psychiatry 7, 199. https://doi.org/10.3389/fpsyt.2016. 00199

Friston, K., 2010. The free-energy principle: a unified brain theory? Nature Reviews. Neuroscience 11, 127-138. https://doi.org/10. 1038/nrn2787

Friston, K., FitzGerald, T., Rigoli, F., Schwartenbeck, P., Pezzulo, G., 2017. Active Inference: A Process Theory. Neural Computation 29, 1-49. https://doi.org/10.1162/NECO a 00912

Friston, K., Rigoli, F., Ognibene, D., Mathys, C., Fitzgerald, T., Pezzulo, G., 2015. Active inference and epistemic value. Cognitive Neuroscience 6, 187-214. https://doi.org/10.1080/17588928.2015. 1020053

Friston, K., Thornton, C., Clark, A., 2012. Free-energy minimization and the dark-room problem. Front Percept Science 3, 130. https://doi. org/10.3389/fpsyg.2012.00130

Friston, K.J., Stephan, K.E., Montague, R., Dolan, R.J., 2014. Computational psychiatry: the brain as a phantastic organ. Lancet Psychiatry 1, 148-158. https://doi.org/10.1016/S2215-0366(14) 70275-5

Froreich, F.V., Vartanian, L.R., Grisham, J.R., Touyz, S.W., 2016. Dimensions of control and their relation to disordered eating behaviours and obsessive-compulsive symptoms. Journal of Eating Disorders 4, 14. https://doi.org/10.1186/s40337-016-0104-4

Gadsby, S., 2017. Distorted body representations in anorexia nervosa. Consciousness and Cognition 51, 17-33. https://doi.org/10.1016/j. concog.2017.02.015

Garfinkel, S.N., Tiley, C., O'Keeffe, S., Harrison, N.A., Seth, A.K., Critchley, H.D., 2016. Discrepancies between dimensions of interoception in autism: Implications for emotion and anxiety. Biological Psychology 114, 117-126. https://doi.org/10.1016/j. biopsycho.2015.12.003

Gorwood, P., Blanchet-Collet, C., Chartrel, N., Duclos, J., Dechelotte, P., Hanachi, M., Fetissov, S., Godart, N., Melchior, J.-C., Ramoz, N., Rovere-Jovene, C., Tolle, V., Viltart, O., Epelbaum, J., 2016. New Insights in Anorexia Nervosa. Frontiers in Neuroscience 10, 256. https://doi.org/10.3389/fnins.2016.00256

Gregertsen, E.C., Mandy, W., Serpell, L., 2017. The Egosyntonic Nature of Anorexia: An Impediment to Recovery in Anorexia Nervosa Treatment. Frontiers in Psychology 8, 2273. https://doi.org/10. 3389/fpsyg.2017.02273

Grzelak, T., Dutkiewicz, A., Paszynska, E., Dmitrzak-Weglarz, M., Slopien, A., Tyszkiewicz-Nwafor, M., 2017. Neurobiochemical and psychological factors influencing the eating behaviors and 
attitudes in anorexia nervosa. - PubMed - NCBI. Journal of Physiology and Biochemistry 73, 297-305. https://doi.org/10. 1007/s13105-016-0540-2

Guidano, V.F., 1987. Complexity of the Self: A Developmental Approach to Psychopathology and Therapy. Guilford Press.

Hamatani, S., Tomotake, M., Takeda, T., Kameoka, N., Kawabata, M., Kubo, H., Ohta, M., Tada, Y., Tomioka, Y., Watanabe, S., Inoshita, M., Kinoshita, M., Ohmori, T., 2018. Impaired central coherence in patients with anorexia nervosa. Psychiatry Research 259, 77-80. https://doi.org/10.1016/j.psychres.2017.09.086

Henningsen, P., Gündel, H., Kop, W.J., Löwe, B., Martin, A., Rief, W., Rosmalen, J.G.M., Schröder, A., van der Feltz-Cornelis, C., Van den Bergh, O., EURONET-SOMA Group, 2018. Persistent Physical Symptoms as Perceptual Dysregulation: A Neuropsychobehavioral Model and Its Clinical Implications. Psychosomatic Medicine 80, 422-431. https://doi.org/10.1097/PSY.0000000000000588

Iodice, P., Porciello, G., Bufalari, I., Barca, L., Pezzulo, G., 2019. An interoceptive illusion of effort induced by false heart-rate feedback. Proceedings of the National Academy of Sciences 201821032. https://doi.org/10.1073/pnas.1821032116

Irvine, K.R., McCarty, K., McKenzie, K.J., Pollet, T.V., Cornelissen, K.K., Tovée, M.J., Cornelissen, P.L., 2019. Distorted body image influences body schema in individuals with negative bodily attitudes. Neuropsychologia 122, 38-50. https://doi.org/10.1016/j. neuropsychologia.2018.11.015

Joffily, M., Coricelli, G., 2013. Emotional valence and the free-energy principle. PLoS Computational Biology 9, e1003094. https://doi. org/10.1371/journal.pcbi.1003094

Kaye, W.H., Wierenga, C.E., Bailer, U.F., Simmons, A.N., BischoffGrethe, A., 2013. Nothing tastes as good as skinny feels: the neurobiology of anorexia nervosa. Trends in Neurosciences 36, 110-120. https://doi.org/10.1016/j.tins.2013.01.003

Keizer, A., Elburg, A. van, Helms, R., Dijkerman, H.C., 2016. A Virtual Reality Full Body Illusion Improves Body Image Disturbance in Anorexia Nervosa. PLoS ONE 11, e0163921. https://doi.org/10. 1371/journal.pone.0163921

Keizer, A., Smeets, M.A.M., Dijkerman, H.C., Uzunbajakau, S.A., van Elburg, A., Postma, A., 2013. Too fat to fit through the door: first evidence for disturbed body-scaled action in anorexia nervosa during locomotion. PLoS One 8, e64602. https://doi.org/10.1371/ journal.pone. 0064602

Keizer, A., Smeets, M.A.M., Dijkerman, H.C., van den Hout, M., Klugkist, I., van Elburg, A., Postma, A., 2011. Tactile body image disturbance in anorexia nervosa. Psychiatry Research 190, 115-120. https://doi.org/10.1016/j.psychres.2011.04.031

Kesby, A., Maguire, S., Brownlow, R., Grisham, J.R., 2017. Intolerance of Uncertainty in eating disorders: An update on the field. Clinical Psychology Review 56, 94-105. https://doi.org/10.1016/j.cpr.2017. 07.002

Kessler, H., Schwarze, M., Filipic, S., Traue, H.C., von Wietersheim, J., 2006. Alexithymia and facial emotion recognition in patients with eating disorders. The International Journal of Eating Disorders 39, 245-251.

Khalsa, S.S., Hassanpour, M.S., Strober, M., Craske, M.G., Arevian, A.C., Feusner, J.D., 2018. Interoceptive Anxiety and Body Representation in Anorexia Nervosa. Frontiers in Psychiatry 9. https://doi.org/10.3389/fpsyt.2018.00444

Klabunde, M., Collado, D., Bohon, C., 2017. An interoceptive model of bulimia nervosa: A neurobiological systematic review. Journal of Psychiatric Research 94, 36-46. https://doi.org/10.1016/j. jpsychires.2017.06.009

Ladouceur, R., Gosselin, P., Dugas, M.J., 2000. Experimental manipulation of intolerance of uncertainty: a study of a theoretical model of worry. Behaviour Research and Therapy 38, 933-941. https://doi. org/10.1016/S0005-7967(99)00133-3
Leppanen, J., Dapelo, M.M., Davies, H., Lang, K., Treasure, J., Tchanturia, K., 2017. Computerised analysis of facial emotion expression in eating disorders. PLoS ONE 12, e0178972. https://doi. org/10.1371/journal.pone.0178972

MacCormack, J.K., Muscatell, K.A., 2019. The metabolic mind: A role for leptin and ghrelin in affect and social cognition. Social and Personality Psychology Compass https://doi.org/10.1111/spc3. 12496

Mahoney, M., Granvold, D.K., 2005. Constructivism and psychotherapy. World Psychiatry 4, 74-77.

Monti, A., Porciello, G., Tieri, G., Aglioti, S.M., 2019. "Embreathment" illusion reveals hierarchical influence of respiratory, visual and spatial signals on corporeal awareness. 10.31234/osf.io/cn9s3

Murphy, J., Brewer, R., Catmur, C., Bird, G., 2017. Interoception and psychopathology: A developmental neuroscience perspective. Developmental Cognitive Neuroscience 23, 45-56. https://doi.org/ 10.1016/j.den.2016.12.006

National Collaborating Centre for Mental Health (UK), 2004. Eating Disorders: Core Interventions in the Treatment and Management of Anorexia Nervosa, Bulimia Nervosa and Related Eating Disorders, National Institute for Health and Clinical Excellence: Guidance. British Psychological Society (UK), Leicester (UK).

Nordbø, R. H. S., Espeset, E. M. S., Gulliksen, K. S., Skårderud, F., Holte, A., 2006. The meaning of self-starvation: Qualitative study of patients' perception of anorexia nervosa. Int J Eat Disord 39, 556 564. https://doi.org/10.1002/eat.20276

Nummenmaa, L., Glerean, E., Hari, R., Hietanen, J.K., 2014. Bodily maps of emotions. Proceedings of the National Academy of Sciences of the United States of America 111, 646-651. https:// doi.org/10.1073/pnas.1321664111

Nunn, K., Frampton, I., Fuglset, T.S., Törzsök-Sonnevend, M., Lask, B. 2011. Anorexia nervosa and the insula. Medical Hypotheses 76, 353-357. https://doi.org/10.1016/j.mehy.2010.10.038

Parr, T., Friston, K.J., 2017. Working memory, attention, and salience in active inference. Scientific Reports 7, 14678.

Paulus, M.P., 2019. Driven by Pain, Not Gain: Computational Approaches to Aversion-Related Decision Making in Psychiatry. Biological Psychiatry. https://doi.org/10.1016/j.biopsych.2019.08. 025

Paulus, M.P., Feinstein, J.S., Khalsa, S.S., 2019a. An Active Inference Approach to Interoceptive Psychopathology. Annual Review of Clinical Psychology 15, 97-122. https://doi.org/10.1146/annurevclinpsy-050718-095617

Paulus, M.P., Feinstein, J.S., Khalsa, S.S., 2019b. An Active Inference Approach to Interoceptive Psychopathology. Annual Review of Clinical Psychology 15, 97-122. https://doi.org/10.1146/annurevclinpsy-050718-095617

Petersen, S., Schroijen, M., Mölders, C., Zenker, S., Van den Bergh, O., 2014. Categorical interoception: perceptual organization of sensations from inside. Psychological Science 25, 1059-1066. https://doi. org/10.1177/0956797613519110

Pezzulo, G., 2013. Why do you fear the Bogeyman? An embodied predictive coding model of perceptual inference. Cognitive, Affective, \& Behavioral Neuroscience

Pezzulo, G., Barca, L., Friston, K.J., 2015a. Active inference and cognitive-emotional interactions in the brain. The Behavioral and Brain Sciences 38, e85. https://doi.org/10.1017/ S0140525X14001009

Pezzulo, G., Iodice, P., Barca, L., Chausse, P., Monceau, S., Mermillod, M., 2018a. Increased heart rate after exercise facilitates the processing of fearful but not disgusted faces. Scientific Reports 8, 398. https://doi.org/10.1038/s41598-017-18761-5

Pezzulo, G., Maisto, D., Barca, L., Van den Bergh, O., 2019a. Perception and misperception of bodily symptoms from an Active Inference perspective: Modelling the case of panic disorder. (preprint). Open Science Framework. https://oi.org/10.31219/osf.io/dywfs 
Pezzulo, G., Maisto, D., Barca, L., Van den Bergh, O., 2019b. Symptom Perception From a Predictive Processing Perspective. Clinical Psychology in Europe e35952.

Pezzulo, G., Rigoli, F., Friston, K., 2018b. Hierarchical Active Inference: A Theory of Motivated Control. Trends in Cognitive Sciences 22, 294-306. https://doi.org/10.1016/j.tics.2018.01.009

Pezzulo, G., Rigoli, F., Friston, K.J., 2015b. Active Inference, homeostatic regulation and adaptive behavioural control. Progress in Neurobiology

Pollatos, O., Herbert, B.M., Mai, S., Kammer, T., 2016. Changes in interoceptive processes following brain stimulation. Philosophical Transactions of the Royal Society of London. Series B, Biological Sciences 371. https://doi.org/10.1098/rstb.2016.0016

Pollatos, O., Kurz, A.-L., Albrecht, J., Schreder, T., Kleemann, A.M., Schöpf, V., Kopietz, R., Wiesmann, M., Schandry, R., 2008. Reduced perception of bodily signals in anorexia nervosa. Eating Behaviors 9, 381-388. https://doi.org/10.1016/j.eatbeh.2008.02.001

Powers, A.R., Mathys, C., Corlett, P.R., 2017. Pavlovian conditioninginduced hallucinations result from overweighting of perceptual priors. Science 357, 596-600. https://doi.org/10.1126/science. aan3458

Quadt, L., Critchley, H.D., Garfinkel, S.N., 2018. The neurobiology of interoception in health and disease. Annals of the New York Academy of Sciences 1428, 112-128. https://doi.org/10.1111/nyas. 13915

Riva, G., Gaudio, S., 2018. Locked to a wrong body: Eating disorders as the outcome of a primary disturbance in multisensory body integration. Consciousness and Cognition 59, 57-59. https://doi.org/10. 1016/j.concog.2017.08.006

Rogers-Carter, M.M., Christianson, J.P., 2019. An insular view of the social decision-making network. Neuroscience and Biobehavioral Reviews 103, 119-132. https://doi.org/10.1016/j.neubiorev.2019. 06.005

Sagliano, L., Magliacano, A., Parazzini, M., Fiocchi, S., Trojano, L., Grossi, D., 2019. Modulating interoception by insula stimulation: A double-blinded tDCS study. Neuroscience Letters 696, 108-113. https://doi.org/10.1016/j.neulet.2018.12.022

Schalla, M.A., Stengel, A., 2017. The Role of Ghrelin in Anorexia Nervosa. International Journal of Molecular Sciences 1-16. doi: https://doi.org/10.3390/ijms19072117

Seth, A.K., 2013. Interoceptive inference, emotion, and the embodied self. Trends in Cognitive Sciences https://doi.org/10.1016/j.tics. 2013.09.007

Seth, A.K., Friston, K.J., 2016. Active interoceptive inference and the emotional brain. Philosophical Transactions of the Royal Society of London. Series B, Biological Sciences 371. https://doi.org/10. 1098/rstb.2016.0007

Seth, A.K., Suzuki, K., Critchley, H.D., 2012. An Interoceptive Predictive Coding Model of Conscious Presence. Frontiers in Psychology 2. https://doi.org/10.3389/fpsyg.2011.00395

Seth, A.K., Tsakiris, M., 2018. Being a Beast Machine: The Somatic Basis of Selfhood. Trends in Cognitive Sciences 22, 969-981. https://doi.org/10.1016/j.tics.2018.08.008

Shihata, S., McEvoy, P.M., Mullan, B.A., Carleton, R.N., 2016. Intolerance of uncertainty in emotional disorders: What uncertainties remain? Journal of Anxiety Disorders 41, 115-124. https:// doi.org/10.1016/j.janxdis.2016.05.001

Spitoni, G.F., Serino, A., Cotugno, A., Mancini, F., Antonucci, G., Pizzamiglio, L., 2015. The two dimensions of the body representation in women suffering from Anorexia Nervosa. Psychiatry Research 230, 181-188. https://doi.org/10.1016/j.psychres.2015. 08.036

Stephan, K.E., Manjaly, Z.M., Mathys, C.D., Weber, L.A.E., Paliwal, S., Gard, T., Tittgemeyer, M., Fleming, S.M., Haker, H., Seth, A.K., Petzschner, F.H., 2016. Allostatic Self-efficacy: A Metacognitive Theory of Dyshomeostasis-Induced Fatigue and Depression.
Frontiers in Human Neuroscience 10. https://doi.org/10.3389/ fnhum.2016.00550

Sterling, P., 2012. Allostasis: a model of predictive regulation. Physiology \& Behavior 106, 5-15.

Sternheim, L., Startup, H., Schmidt, U., 2011. An experimental exploration of behavioral and cognitive-emotional aspects of intolerance of uncertainty in eating disorder patients. Journal of Anxiety Disorders 25, 806-812. https://doi.org/10.1016/j.janxdis.2011.03.020

Sterzer, P., Adams, R.A., Fletcher, P., Frith, C., Lawrie, S.M., Muckli, L., Petrovic, P., Uhlhaas, P., Voss, M., Corlett, P.R., 2018. The Predictive Coding Account of Psychosis. Biological Psychiatry 84, 634-643. https://doi.org/10.1016/j.biopsych.2018.05.015

Strigo, I.A., Matthews, S.C., Simmons, A.N., Oberndorfer, T., Klabunde, M., Reinhardt, L.E., Kaye, W.H., 2013. Altered insula activation during pain anticipation in individuals recovered from anorexia nervosa: evidence of interoceptive dysregulation. The International Journal of Eating Disorders 46, 23-33. https://doi.org/10.1002/eat. 22045

Surgenor, L.J., Horn, J., Plumridge, E.W., Hudson, S.M., 2002. Anorexia nervosa and psychological control: a reexamination of selected theoretical accounts. European Eating Disorders Review 10, 85-101. https://doi.org/10.1002/erv.457

Tajadura-Jiménez, A., Tsakiris, M., 2014. Balancing the "inner" and the "outer" self: Interoceptive sensitivity modulates self-other boundaries. Journal of Experimental Psychology. General 143, 736-744. https://doi.org/10.1037/a0033171

Tschantz, A., Seth, A.K., Buckley, C.L., 2019. Learning action-oriented models through active inference. bioRxiv 764969. https://doi.org/ $10.1101 / 764969$

Van den Bergh, O., Witthöft, M., Petersen, S., Brown, R.J., 2017. Symptoms and the body: Taking the inferential leap. Neuroscience and Biobehavioral Reviews 74, 185-203. https://doi.org/10.1016/j. neubiorev.2017.01.015

Watson, H.J., Yilmaz, Z., Thornton, L.M., Hübel, C., Coleman, J.R.I., Gaspar, H.A., Bryois, J., Hinney, A., Leppä, V.M., Mattheisen, M., Medland, S.E., Ripke, S., Yao, S., Giusti-Rodríguez, P., Hanscombe, K.B., Purves, K.L., Adan, R.A.H., Alfredsson, L., Ando, T., Andreassen, O.A., Baker, J.H., Berrettini, W.H., Boehm, I., Boni, C., Perica, V.B., Buehren, K., Burghardt, R., Cassina, M., Cichon, S., Clementi, M., Cone, R.D., Courtet, P., Crow, S., Crowley, J.J., Danner, U.N., Davis, O.S.P., Zwaan, M. de, Dedoussis, G., Degortes, D., DeSocio, J.E., Dick, D.M., Dikeos, D., Dina, C., Dmitrzak-Weglarz, M., Docampo, E., Duncan, L.E., Egberts, K., Ehrlich, S., Escaramís, G., Esko, T., Estivill, X., Farmer, A., Favaro, A., Fernández-Aranda, F., Fichter, M.M., Fischer, K., Föcker, M., Foretova, L., Forstner, A.J., Forzan, M., Franklin, C.S., Gallinger, S., Giegling, I., Giuranna, J., Gonidakis, F., Gorwood, P., Mayora, M.G., Guillaume, S., Guo, Y., Hakonarson, H., Hatzikotoulas, K., Hauser, J., Hebebrand, J., Helder, S.G., Herms, S., Herpertz-Dahlmann, B., Herzog, W., Huckins, L.M., Hudson, J.I., Imgart, H., Inoko, H., Janout, V., Jiménez-Murcia, S., Julià, A., Kalsi, G., Kaminská, D., Kaprio, J., Karhunen, L., Karwautz, A., Kas, M.J.H., Kennedy, J.L., Keski-Rahkonen, A., Kiezebrink, K., Kim, Y.-R., Klareskog, L., Klump, K.L., Knudsen, G.P.S., Via, M.C.L., Hellard, S.L., Levitan, R.D., Li, D., Lilenfeld, L., Lin, B.D., Lissowska, J., Luykx, J., Magistretti, P.J., Maj, M., Mannik, K., Marsal, S., Marshall, C.R., Mattingsdal, M., McDevitt, S., McGuffin, P., Metspalu, A., Meulenbelt, I., Micali, N., Mitchell, K., Monteleone, A.M., Monteleone, P., Munn-Chernoff, M.A., Nacmias, B., Navratilova, M., Ntalla, I., O'Toole, J.K., Ophoff, R.A., Padyukov, L., Palotie, A., Pantel, J., Papezova, H., Pinto, D., Rabionet, R., Raevuori, A., Ramoz, N., Reichborn-Kjennerud, T., Ricca, V., Ripatti, S., Ritschel, F., Roberts, M., Rotondo, A., Rujescu, D., Rybakowski, F., Santonastaso, P., Scherag, A., Scherer, S.W., Schmidt, U., Schork, N.J., Schosser, A., Seitz, J., Slachtova, L., Slagboom, P.E., Landt, M.C.T.S.-O. 't, Slopien, A., 
Sorbi, S., Świątkowska, B., Szatkiewicz, J.P., Tachmazidou, I., Tenconi, E., Tortorella, A., Tozzi, F., Treasure, J., Tsitsika, A., Tyszkiewicz-Nwafor, M., Tziouvas, K., van Elburg, A.A., van Furth, E.F., Wagner, G., Walton, E., Widen, E., Zeggini, E., Zerwas, S., Zipfel, S., Bergen, A.W., Boden, J.M., Brandt, H., Crawford, S., Halmi, K.A., Horwood, L.J., Johnson, C., Kaplan, A.S., Kaye, W.H., Mitchell, J.E., Olsen, C.M., Pearson, J.F., Pedersen, N.L., Strober, M., Werge, T., Whiteman, D.C., Woodside, D.B., Stuber, G.D., Gordon, S., Grove, J., Henders, A.K., Juréus, A., Kirk, K.M., Larsen, J.T., Parker, R., Petersen, L., Jordan, J., Kennedy, M., Montgomery, G.W., Wade, T.D., Birgegård, A., Lichtenstein, P., Norring, C., Landén, M., Martin, N.G., Mortensen, P.B., Sullivan, P.F., Breen, G., Bulik, C.M., 2019. Genome-wide association study identifies eight risk loci and implicates metabo-psychiatric origins for anorexia nervosa. Nature Genetics 1. https://doi.org/10.1038/s41588-019-0439-2

Wu, J. T., Kral, J. G., 2004. Ghrelin. Integrative neuroendocrine peptide in health and disease. Annals of Surgery 4, 464-74.
Young, H.A., Gaylor, C.M., de Kerckhove, D., Watkins, H., Benton, D., 2019. Interoceptive accuracy moderates the response to a glucose load: a test of the predictive coding framework. Proceedings of the Royal Society B: Biological Sciences 286, 20190244. https://doi. org/10.1098/rspb.2019.0244

Young, H.A., Williams, C., Pink, A.E., Freegard, G., Owens, A., Benton, D., 2017. Getting to the heart of the matter: Does aberrant interoceptive processing contribute towards emotional eating? PLoS ONE 12, e0186312. https://doi.org/10.1371/journal.pone.0186312

Yu, A.A.N., Iodice, P., Pezzulo, G., Barca, L. (2020, January 29). Bodily Information and Top-Down Affective Priming Jointly Affect the Processing of Fearful Faces. Preprint retrieved from https://doi.org/ 10.31234/osf.io/h76a9

Publisher's note Springer Nature remains neutral with regard to jurisdictional claims in published maps and institutional affiliations. 